\title{
Robust Optimization Utilizing the Second-Order Design Sensitivity Information
}

\author{
Nam-Kyung Kim ${ }^{1}$, Dong-Hun Kim ${ }^{1}$, Dong-Wook Kim ${ }^{1}$, Heung-Geun Kim ${ }^{1}$, David A. Lowther ${ }^{2}$, and \\ Jan K. Sykulski ${ }^{3}$, Fellow, IEEE \\ ${ }^{1}$ School of Electrical Engineering and Computer Science, Kyungpook National University, Daegu 702-701, Korea \\ 2 Electrical and Computer Engineering Department, McGill University, Montreal, QC H3A 2A7, Canada \\ ${ }^{3}$ School of Electronics and Computer Science, University of Southampton, Southampton SO17 1BJ, U.K.
}

\begin{abstract}
This paper presents an effective methodology for robust optimization of electromagnetic devices. To achieve the goal, the method improves the robustness of the minimum of the objective function chosen as a design solution by minimizing the second-order sensitivity information, called a gradient index (GI) and defined by a function of gradients of performance functions with respect to uncertain variables. The constraint feasibility is also enhanced by adding a GI corresponding to the constraint value. The distinctive feature of the method is that it requires neither statistical information on design variables nor calculation of the performance reliability during the robust optimization process. The validity of the proposed method is tested with the TEAM Workshop Problem 22.
\end{abstract}

Index Terms-Optimization, robust optimization, sensitivity analysis.

\section{INTRODUCTION}

D UE to a growing demand for high-performance and high-reliability electromagnetic devices or equipment, attention has recently focused on the robust optimization of products with the aim of minimizing the variation of the performance as a result of uncontrollable factors such as manufacturing errors, operating conditions, material properties, etc. [1]-[5]. Until now, most of the reported attempts have used the Taguchi's robust design concept or Monte Carlo simulation based on the assumption that design parameters are random variables with a probability distribution [2]-[4]. However, implementation difficulties usually arise because it is not easy to acquire probability data of uncertain variables; moreover, information about which parameter is dominant may not be available.

To overcome the aforementioned drawbacks, this paper proposes an effective methodology utilizing the second-order sensitivity information, defined as a "gradient index" (GI) in [5], for the robust optimization of electromagnetic systems. The basic concept of the method is to obtain robustness of the objective function by minimizing a GI value calculated from the gradients of performance functions with respect to uncertain variables. Simultaneously, the constraint feasibility is also considered by adding a term determined with a constraint value and a GI corresponding to the constraint. Consequently, the method needs neither statistical information on design variations nor calculation of the performance reliability while it is searching for a robust optimal solution.

In this paper, the optimization method is numerically implemented by combining the commercial finite element code MagNet [6] with a Design Optimization Tools (DOT) optimizer [7]. A modified feasible direction algorithm with the secondorder sensitivity information by finite differencing is used to

Manuscript received December 11, 2009; accepted February 10, 2010. Current version published July 21, 2010. Corresponding author: D.-H. Kim (e-mail: dh29kim@ee.knu.ac.kr).

Color versions of one or more of the figures in this paper are available online at http://ieeexplore.ieee.org.

Digital Object Identifier 10.1109/TMAG.2010.2043719 obtain a robust optimal solution. The validity of the proposed method is tested with the TEAM Workshop Problem 22.

\section{BASIC CONCEPTS OF A GRADIENT INDEX FORMULATION}

To help designers understand the GI formulation presented by Han and Kwak [5] in mechanical engineering, its basic concepts are briefly summarized.

A typical optimization problem with the aim of minimizing an objective/performance function $f$, subject to a set of constrains $g_{j}$, is expressed as

$$
\begin{aligned}
\text { Minimize } & f(\mathbf{x}) \\
\text { subject to } & g_{j}(\mathbf{x}) \leq 0, \quad j=1,2, \ldots m \\
& \mathbf{x}_{\mathrm{L}} \leq \mathbf{x} \leq \mathbf{x}_{\mathrm{U}}
\end{aligned}
$$

where $\mathbf{x}$ is an $n$-dimensional design variable vector. The values $\mathbf{x}_{\mathrm{L}}$ and $\mathbf{x}_{\mathrm{U}}$ denote the lower and upper bounds of the design variable vector, respectively. Conventional optimization methods do not take into account the effect of variations in design variables on the objective function and the constraint feasibility. Thus, an optimum solution obtained might be very sensitive, or the constraints might be violated, in the presence of perturbations of the design variables.

With the aim to improve the robustness of the objective and constraint functions effectively, the proposed robust optimization method in essence utilizes the GI value, defined as a maximum gradient of the objective function of interest with respect to uncertain variables, $u_{i}$, as follows:

$$
\mathrm{GI}=\max _{i}\left|d f / d u_{i}\right|, \quad i=1,2, \ldots, N, \quad u_{i} \in \mathbf{x}
$$

where $N$ denotes the number of uncertain variables considered in the robust optimization problem. The robustness of the objective function as well as constraint feasibility are simultaneously improved by searching for a new optimum solution of the robust optimization problem formulated with the concept of GI as follows:

$$
\begin{aligned}
\text { Minimize } & \mathrm{GI}_{f}=\max _{i}\left|d f(\mathbf{x}) / d u_{i}\right|, \quad i=1,2, \ldots N \\
\text { subject to } & g_{j}(\mathbf{x})+\Psi_{j}\left(g_{j}(\mathbf{x})\right) \leq 0, \quad j=1,2, \ldots m \\
& f(\mathbf{x}) \cong M
\end{aligned}
$$




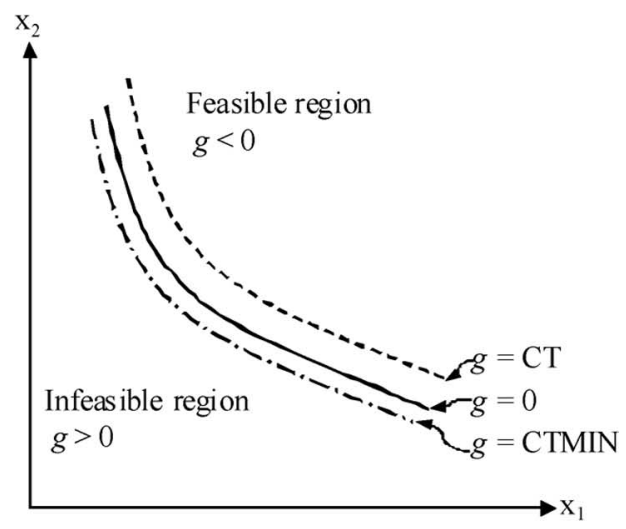

(a)

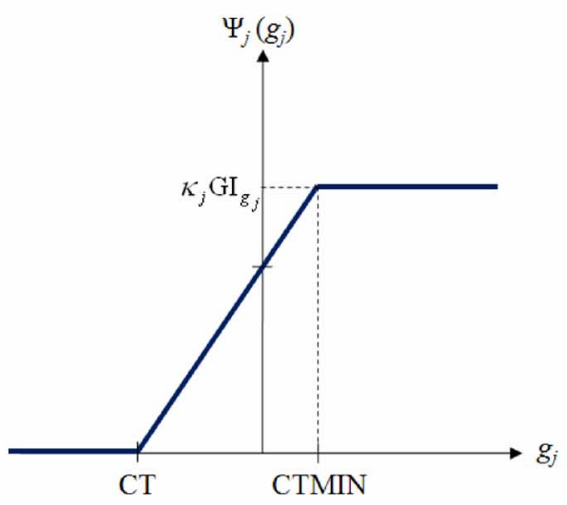

(b)

Fig. 1. Illustration of geometric relationship between $g$, CT, CTMIN, and $\Psi$. (a) Constraint function $g$. (b) Penalty function $\Psi_{j}\left(g_{j}\right)$.

where $\mathrm{GI}_{f}$ is a gradient function of the objective function with respect to the uncertain variables $u_{i}$, and $M$ is a target value of the objective function determined by a designer after solving (1). In order to enhance the robustness of constraint feasibility, an additional term, $\Psi_{j}\left(g_{j}\right)$, is added to each constraint function in (3). The penalty function can be defined as

$$
\begin{aligned}
\Psi_{j}\left(g_{j}\right) & = \begin{cases}0, & g_{j}<\mathrm{CT} \\
\frac{\kappa_{j} \mathrm{GI}_{g_{j}}}{\mathrm{CTMIN}_{\mathrm{TMT}},}, & \mathrm{CT}<g_{j}<\mathrm{CTMIN} \\
\kappa_{j} \mathrm{GI}_{g_{j}}, & g_{j}>\mathrm{CTMIN}\end{cases} \\
\mathrm{GI}_{g_{j}} & =\max _{i}\left|d g_{j}(\mathbf{x}) / d u_{i}\right|, \quad i=1,2, \ldots N
\end{aligned}
$$

where $\mathrm{GI}_{g_{j}}$ is a gradient function of the $j$ th constraint function with respect to the uncertain variables. The values CT and CTMIN are constraint tolerances, respectively, as illustrated in Fig. 1, while the factor $\kappa$ is selected depending on the importance of the constraint and the number of design variations from the viewpoint of robustness. When the constraint is in a feasible region $\left(g_{j}<\mathrm{CT}\right)$, the penalty term is zero. In the case that the constraint is numerically critical (CT $\leq g_{j} \leq \mathrm{CTMIN}$ ) or violated $\left(g_{j}>\mathrm{CTMIN}\right)$, the penalty term is proportional to the gradient index for the constraint function and $\kappa$ is added according to the value of $g_{j}$ for the robustness of the constraint feasibility.

Since the gradient index is incorporated with both the objective function and the constraints to increase the design robustness, robust designs can be effectively obtained.

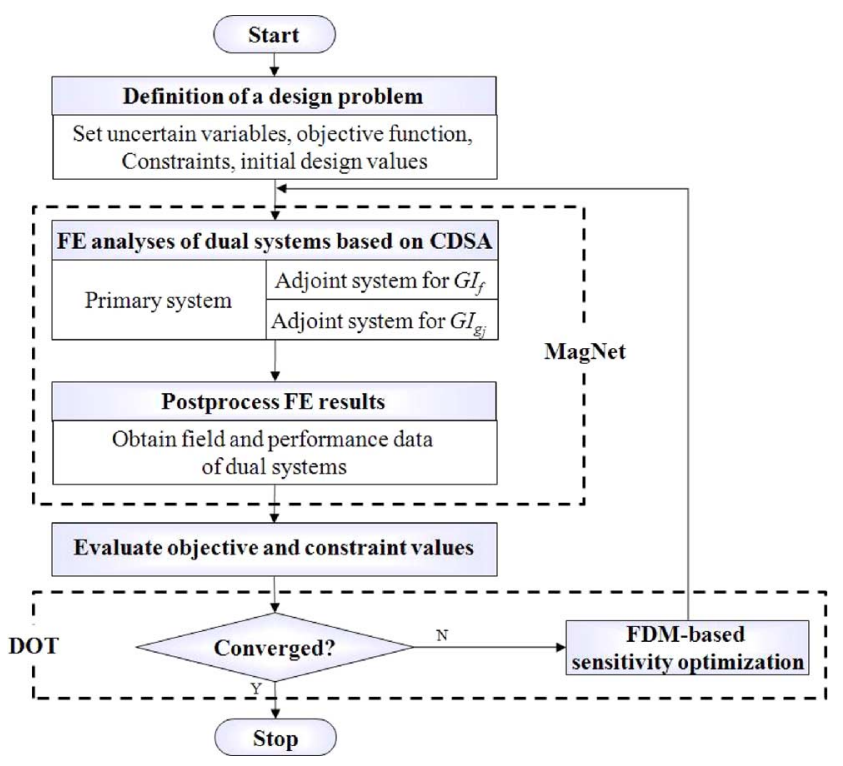

Fig. 2. Flowchart of the proposed robust optimization method.

\section{NUMERICAL IMPLEMENTATION}

The proposed method has been implemented by combining the commercial finite element code MagNet with a DOT optimizer as shown in Fig. 2, where a modified feasible direction algorithm with the second-order sensitivity information by finite differencing is used to search for a robust optimal solution. To obtain the GI values, the first-order sensitivity values are computed by the continuum design sensitivity analysis (CDSA) [8], [9], and then the second-order sensitivity information on the objective function with respect to the selected uncertain variables is calculated based on the finite difference technique in the DOT optimizer.

If a conventional optimization is carried out prior to robust optimization, designers can determine an achievable range of the objective function and also obtain other useful information, such as about which design variables are most sensitive to change during the optimization and which constraint is significant in the problem considered. Assuming that such useful references are available through the deterministic optimization, the proposed algorithm for robust optimization of electromagnetic devices consists of the following steps.

1. Define an objective function, uncertain variables, and constraint functions and formulate a robust optimization problem with (3).

2. Solve the dual system for the objective function based on CDSA and-if necessary-solve additional dual systems for constraint functions.

3. Extract field and performance data of interest from postprocessed analysis data.

4. Compute the values relating to the objective function, constraints, and accordingly their sensitivities.

5. If the design is acceptable for the prescribed convergence criteria, then the optimization process stops.

6. Otherwise, calculate the second-order gradients of objective and constraint functions by the finite difference method and go to step 2 .

In comparison to previous approaches for robust optimal design, such as Taguchi's robust design concept or Monte Carlo 


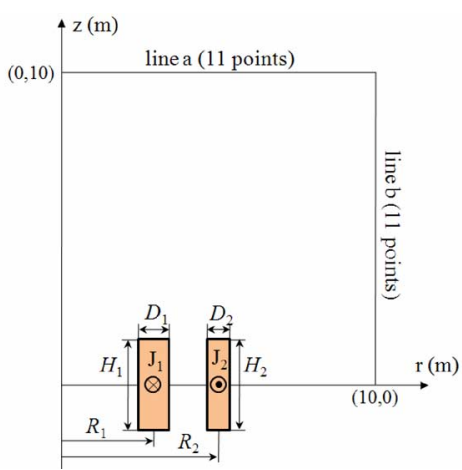

Fig. 3. Configuration of the SMES device with eight design variables.

simulation, which require statistical information on design variations and calculate the performance reliability, the method based on the gradient index needs no such information.

\section{RESULTS}

The TEAM benchmark problem 22 is concerned with the design optimization of a superconducting magnetic energy storage system (SMES) as depicted in Fig. 3 [3], [4]. In order to simplify the design problem, a constraint of the current quench condition on the superconductivity magnet is not considered here. A typical optimization problem for minimizing an objective function subject to a set of constraints is expressed as

$$
\begin{array}{cl}
\text { Minimize } & f(\mathbf{x})=\sum_{i=1}^{21}\left|B_{\text {stray }, i}(\mathbf{x})\right|^{2} \\
\text { subject to } & g_{1}(\mathbf{x})=\left(\frac{E(\mathbf{x})-E_{o}}{0.05 \times E_{o}}\right)^{2}-1 \leq 0 \\
& g_{2}=\left(R_{1}-R_{2}\right)+\frac{1}{2}\left(D_{1}+D_{2}\right) \leq 0 \\
& \mathbf{x}_{L} \leq \mathbf{x} \leq \mathbf{x}_{U}
\end{array}
$$

where $B_{\text {stray }, i}$ is the stray field values calculated at the $i$ th measurement point along line a and line b, $E$ is the stored magnetic energy, and $E_{o}$ is the energy target value of $180 \mathrm{MJ}$. The design variable vector $\mathbf{x}$ consists of six parameters describing the dimensions of the magnet and the two current densities. To search for an optimal solution of (5) based on CDSA, two dual systems for the objective function $f(\mathbf{x})$ and the constraint function $g_{1}(\mathbf{x})$, which are implicit functions with respect to design variables, should be constructed. However, there is no need to analyze the adjoint system in the case of $g_{1}(\mathbf{x})$ because the dual system itself is self-adjoint [8], [9].

To complement the above expressions, the proposed robust optimization for improving the robustness of the objective and the constraint functions is formulated as follows:

$$
\begin{array}{cl}
\text { Minimize } & \mathrm{GI}_{f}=\max _{i}\left|d f(\mathbf{x}) / d u_{i}\right|, \quad i=1,2, \ldots 8 \\
\text { subject to } & g_{j}(\mathbf{x})+\Psi_{j}\left(g_{j}(\mathbf{x})\right) \leq 0, \quad j=1,2 \\
& f(\mathbf{x}) \cong M \\
& \mathrm{GI}_{g_{j}}=\max _{i}\left|d g_{j}(\mathbf{x}) / d u_{i}\right|, \quad i=1,2, \ldots 8
\end{array}
$$

where $M$ is set to be the same as the energy target value $E_{o}$. In order to enhance the robustness of the constraint feasibility, the penalty function $\Psi_{j}$ with $\kappa_{1}=1$ and $\kappa_{2}=2$ shown in (4) is

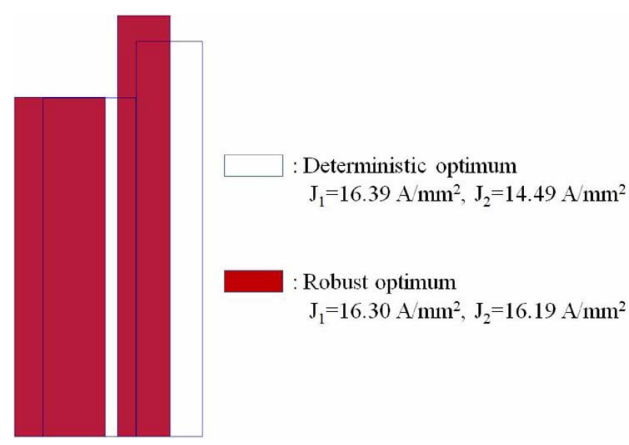

Fig. 4. Comparison of magnet dimensions after optimization.

TABLE I

DESIGN VARIABLES AND PERFORMANCE INDICATIONS AT THE DETERMINISTIC AND ROBUST OPTIMA

\begin{tabular}{|c|c|c|c|c|c|c|}
\hline $\begin{array}{c}\text { Design } \\
\text { variables }\end{array}$ & Unit & $\begin{array}{c}\text { Lower } \\
\text { bound } \\
\left(\mathbf{x}_{L}\right)\end{array}$ & $\begin{array}{c}\text { Initial } \\
\text { values }\end{array}$ & $\begin{array}{c}\text { CDSA } \\
\text { optimum }\end{array}$ & $\begin{array}{c}\text { Robust } \\
\text { optimum }\end{array}$ & $\begin{array}{c}\text { Upper } \\
\text { bound } \\
\left(\mathbf{x}_{U}\right)\end{array}$ \\
\hline $\mathrm{R} 1$ & $\mathrm{~mm}$ & 1000 & 2000 & 2108 & 1977 & 4000 \\
\hline $\mathrm{D} 1$ & $\mathrm{~mm}$ & 100 & 500 & 412 & 404 & 800 \\
\hline $\mathrm{H} 1$ & $\mathrm{~mm}$ & 200 & 1500 & 1504 & 1507 & 3600 \\
\hline $\mathrm{R} 2$ & $\mathrm{~mm}$ & 1800 & 2500 & 2462 & 2348 & 5000 \\
\hline $\mathrm{D} 2$ & $\mathrm{~mm}$ & 100 & 400 & 294 & 233 & 800 \\
\hline $\mathrm{H} 2$ & $\mathrm{~mm}$ & 200 & 2000 & 1756 & 1871 & 3600 \\
\hline $\mathrm{J} 1$ & $\mathrm{~A} / \mathrm{mm}^{2}$ & 10 & 17 & 16.39 & 16.30 & 30 \\
\hline $\mathrm{J} 2$ & $\mathrm{~A} / \mathrm{mm}^{2}$ & 10 & 17 & 14.49 & 16.19 & 30 \\
\hline $\mathrm{B}_{\text {stray,mean }}$ & $\mu \mathrm{T}$ & & 23,055 & 153 & 34 & \\
\hline Energy & $\mathrm{MJ}$ & & 521 & 183 & 181 & \\
\hline
\end{tabular}

taken into account, and the default values of $\mathrm{CT}=-0.03$ and $\mathrm{CTMIN}=0.003$ provided by the DOT optimizer are allotted to the constraint tolerances.

The optimization problem for minimizing the stray fields of the SMES device of Fig. 3 is solved using two methods. The first is a deterministic method based on CDSA that does not take into account the effects of uncertain parameters; the second approach is the proposed robust optimization method. In this paper, all of the eight design variables used in the deterministic method are selected to be the uncertain variables for the purpose of comparison between the two methods.

Starting with an initial design, the deterministic and the robust optima are presented in Table I. The stored energy values obtained from the two methods almost reach the target value of $180 \mathrm{MJ}$, but the robust optimum produces a better mean value of the stray fields than the deterministic algorithm. It is inferred that the deterministic optimal solution is trapped in one of the local minima near the constraint boundaries, while a better optimal solution is found by the robust optimization as the feasibility robustness of the constraints is improved.

In Fig. 4, the dimensions of the two optimized magnets are compared to each other. As shown in the figure, the distance between the two windings resulting from the deterministic optimization is too small to be fabricated in practice. On the other hand, the robust optimization yields a more acceptable result by prescribing a relatively large value to the factor $\kappa_{2}$ in $g_{2}$. That is proved by Fig. 5, where the more important constraint in terms of $g_{2}$ becomes very robust when the feasibility robustness is considered in the optimization. The comparison of the sensitivity values of the objective function between the deterministic 


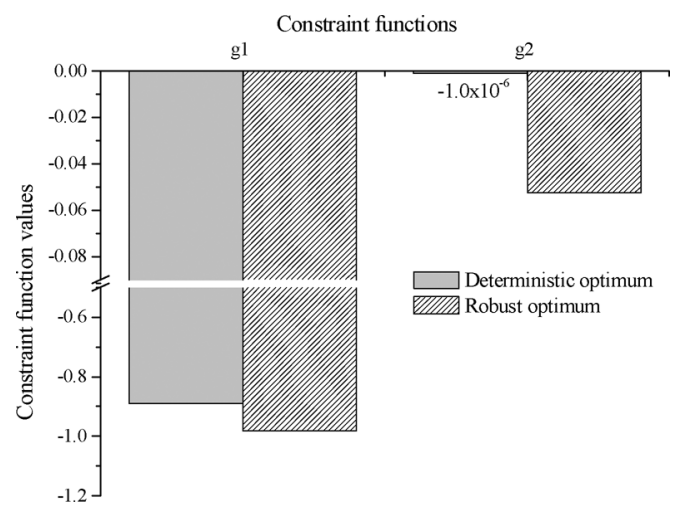

Fig. 5. Comparison of constraint function values after optimization.

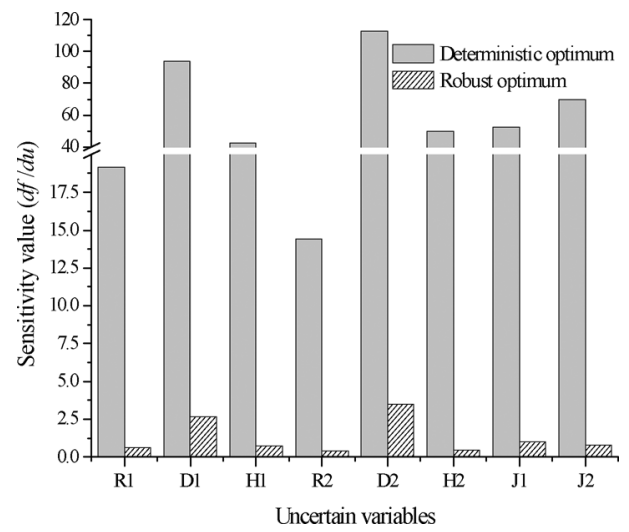

Fig. 6. Comparison of sensitivity values of uncertain variables.

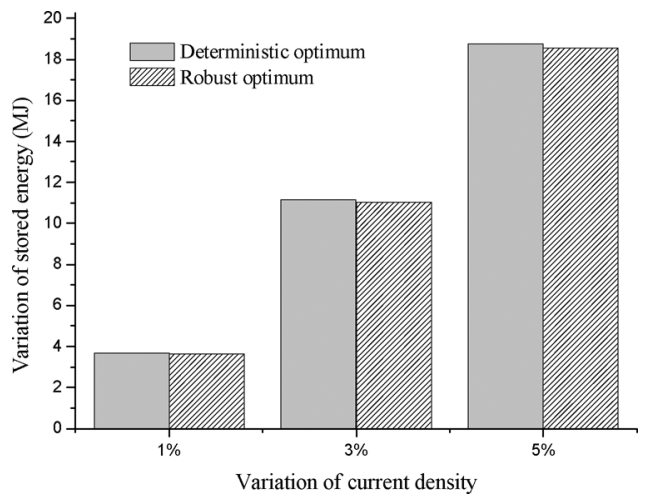

Fig. 7. Comparison of stored energy variations when current density changes.

and the robust optima is presented in Fig. 6, where a large sensitivity value implies that the given design is very sensitive to the design variations. It is noticed that the robust optimum dramatically improves the robustness of the objective function.

In Figs. 7 and 8, the variations of the stored energy values and the stray fields are compared between the deterministic and the robust optima, respectively, when the current density values of $\mathrm{J} 1$ and $\mathrm{J} 2$ change by the amount of $1 \%, 3 \%$, and $5 \%$ of their optimized current values, respectively. These results correspond to the changes of the constraint function $g_{1}$ in (5) and the objective function in a case that the uncertainty may come from a current controller, which can keep the currents within a certain range when compensating perturbations. It is concluded that the variations of the stored energy values are almost the same, but

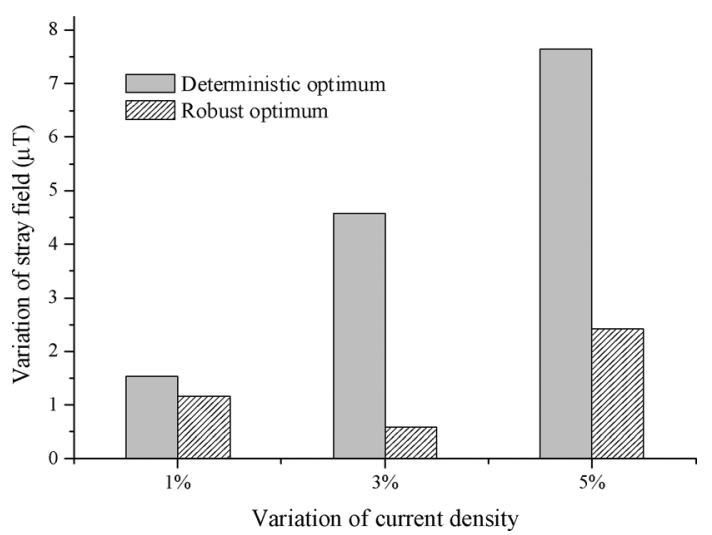

Fig. 8. Comparison of stray field variations when current density changes.

the stray field variations make big differences between the two methods under the condition of small changes of the imposed currents.

\section{CONCLUSION}

In this paper, a robust optimization approach adopting the concept of a gradient index has been described and successfully applied to the robust optimization problem of a superconducting magnetic energy storage system, the TEAM Workshop Problem 22. The results reveal that the proposed method offers high performance as well as robustness of the objective and the constraint functions even without the statistical information on design variations and calculation of the performance reliability during optimization process.

\section{ACKNOWLEDGMENT}

This work has been supported by EIRC (2009T100100496), which is funded by the Ministry of Commerce, Industry and Energy (MOCIE) and Electric Power Industry Technology Evaluation \& Planning (ETEP).

\section{REFERENCES}

[1] B. S. Yoon, I. S. Jung, and D. S. Hyun, "Robust shape optimization of electromagnetic devices," IEEE Trans. Magn., vol. 35, no. 3, pp. 1710-1713, May 1999.

[2] H. T. Wang, Z. J. Liu, S. X. Chen, and J. P. Yang, "Application of Taguchi method to robust design of BLDC motor performance," IEEE Trans. Magn., vol. 35, no. 5, pp. 3700-3702, Sep. 1999.

[3] G. Steiner, A. Weber, and C. Magele, "Managing uncertainties in electromagnetic design problems with robust optimization," IEEE Trans. Magn., vol. 40, no. 2, pp. 1094-1099, Mar. 2004.

[4] F. G. Guimaraes, D. A. Lowther, and J. A. Ramirez, "Multiobjecitve approaches for robust electromagnetic design," IEEE Trans. Magn., vol. 42, no. 4, pp. 1207-1210, Apr. 2006.

[5] J. S. Han and B. M. Kwak, "Robust optimization using a gradient index: MEMS applications," Struct. Multidiscipl. Optim., vol. 27, no. 6, pp. 469-478, 2004.

[6] "MagNet User's Manual," Infolytica Corporation, Quebec, QC, Canada, 2008.

[7] "DOT User Manual," Vanderplaats Research \& Development, Inc., 2001.

[8] D.-H. Kim, K. S. Ship, and J. K. Sykulski, "Applying continuum design sensitivity analysis combined with standard EM software to shape optimization in magnetostatic problems," IEEE Trans. Magn., vol. 40, no. 2, pp. 1156-1159, Mar. 2004

[9] D.-H. Kim, J. K. Sykulski, and D. A. Lowther, "Design optimisation of electromagnetic devices using continuum design sensitivity analysis combined with commercial EM software," IET Trans. Sci., Meas. Technol., vol. 1, no. 1, pp. 30-36, Jan. 2007. 